\title{
Nuevos roles parentales de mediación: percepciones de los padres sobre la relación de sus hijos con múltiples pantallas
}

\section{New parental mediation roles: Parents' perceptions of their children's relationship with multiple screens}

\author{
Rayén Condeza; Nadia Herrada-Hidalgo; Camila Barros-Friz
}

Cómo citar este artículo:

Condeza, Rayén; Herrada-Hidalgo, Nadia; Barros-Friz, Camila (2019). “Nuevos roles parentales de mediación: percepciones de los padres sobre la relación de sus hijos con múltiples pantallas". El profesional de la información, v. 28, n. 4, e280402.

https://doi.org/10.3145/epi.2019.jul.02

Artículo recibido el 11-11-2018

Aceptación definitiva: 28-02-2019

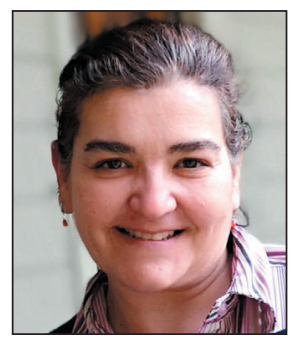

\section{Rayén Condeza $\square$}

https://orcid.org/0000-0003-4517-8585

Pontificia Universidad Católica de Chile Av. Libertador Bernardo O'Higgins, 340. Santiago, Región Metropolitana, Chile rcondeza@uc.cl

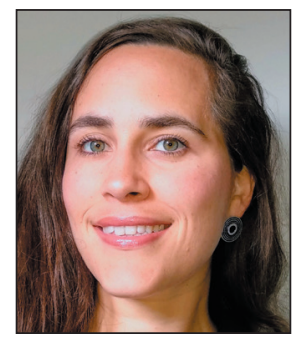

Nadia Herrada-Hidalgo https://orcid.org/0000-0003-4344-6080

Pontificia Universidad Católica de Chile Av. Libertador Bernardo O'Higgins, 340. Santiago, Región Metropolitana, Chile nherrada@uc.cl

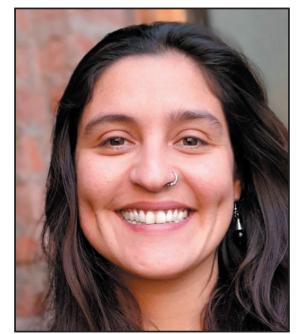

Camila Barros-Friz

https://orcid.org/0000-0003-4212-5234

Pontificia Universidad Católica de Chile Av. Libertador Bernardo O'Higgins, 340. Santiago, Región Metropolitana, Chile cfbarros@uc.cl

\section{Resumen}

En este estudio se difunden los resultados de la primera etapa de una investigación mixta sobre las percepciones que tienen los padres en Chile acerca del uso que hacen sus hijos de entre 8 y 13 años de los medios, en un contexto multipantalla y las prácticas de mediación a las que recurren. A través de un cuestionario auto administrado, se levantó información de base $(n=146)$, como insumo para desarrollar una estrategia de educación digital dirigida a estos adultos, quienes enfrentan el desafío de crianza en un ecosistema distinto al de su propia infancia y para lo cual no han sido preparados. Los resultados muestran la necesidad de la alfabetización transmedia, para que los padres tengan recursos adecuados en el proceso de guiar a sus hijos en la sociedad de la información y en la sociedad red.

\section{Palabras clave}

Mediación parental; Uso de múltiples pantallas; Alfabetización mediática; Alfabetización transmedia; Comunicación; Tecnologías de la información; TIC; Niños; Percepciones; Chile.

\section{Financiación}

Proyecto VRI Inicio 3/2016. Percepciones de padres y profesores sobre consumo y uso de múltiples pantallas. Vicerrectoría de Investigación, Pontificia Universidad Católica de Chile para inicio de carrera de investigación. Investigadora principal: Rayén Condeza. 


\begin{abstract}
This study disseminates the results of the first stage of a mixed investigation on the perceptions that parents have in Chile about the use made by their children between 8 and 13 years of the media, in a multi-screen context and mediation practices to which they resort. Through a self-administered questionnaire, basic information was collected $(n=146)$, as an input to develop a digital education strategy aimed at these adults, who face the challenge of raising an ecosystem different from their own childhood and for which they have not been prepared. The results show the need for transmedia literacy, so that parents have adequate resources to assertively guide the growth of their children in the information society and in the network society.
\end{abstract}

\title{
Keywords
}

Parental mediation; Use of multiple screens; Media literacy; Transmedia literacy; Communication; Information technologies; Children; Perceptions; Chile.

\section{Introducción}

En los últimos años la alfabetización mediática e informacional (MIL) ha sido reconocida por la División de Información y Comunicación de la Unesco como un requisito relevante para fomentar el acceso equitativo de la ciudadanía a la información y al conocimiento y para promover medios de comunicación libres, independientes y pluralistas (Unesco, 2018). Los ciudadanos consumimos medios por doquier y las pantallas se han adueñado de nuestras vidas (Aguaded, 2012). Sin embargo, ¿nos hemos educado para ello? La información y los medios tienen un papel principal en nuestra vida cotidiana (Unesco, 2018). En el ámbito de la crianza los padres se relacionan con sus hijos en hogares multipantalla conectados a internet, lo que implica un desafío sin precedentes para abordar una educación digital (Torrecillas-Lacave; Vásquez-Barrio; Monteagudo-Barandalla, 2017) que les permita ejercer ciudadanía. El mundo al que acceden los niños a través de la Red es diferente al de la propia infancia de los adultos que los están educando, quienes son responsables de diseñar las políticas para asegurar su bienestar (Livingstone; Haddon, 2009), en áreas tan relevantes como la concepción de los derechos a la comunicación de la niñez. Este es un asunto que requiere repensarse hoy en el nuevo escenario tecnológico (Frau-Meiggs, 2012; Livingstone, 2016). Por nuestra parte adoptamos la llamada de atención de Livingstone (2016, citando a Hanson, 2014, p. 4), en cuanto a

"cuidar la investigación que oscurece el examen crítico de las consecuencias intencionales o no de desarrollar legislación, políticas y programas en el nombre de los niños y niñas" (p. 8).

En este contexto, ser padres en la sociedad de la información supone desempeñar nuevos roles de educación medial e informacional. Sin embargo poco sabemos sobre los intereses y competencias con las que los adultos cuentan para cumplir dichos roles. Se puede decir que

"los padres son una pieza clave en el seguimiento que sus hijos destinan al consumo en multipantallas" (Marta-Lazo; Gabelas-Barroso, 2008, p. 111),

Este trabajo aborda la pregunta sobre cuáles son las prácticas de mediación que ejercen los padres en el uso que hacen sus hijos de las TIC y qué percepciones tienen estos adultos sobre las mismas. Nos preguntamos si el perfil tecnológico de los apoderados se relaciona con las prácticas de mediación parental y con dichas percepciones, aspecto que parece necesario abordar para repensar las dinámicas de comunicación en el escenario digital y tecnológico actual.

En este artículo presentamos los resultados de la primera fase de una investigación, de carácter mixta, que se está llevando a cabo en Chile a partir del segundo semestre del año 2016. Su propósito es levantar evidencia de base para obtener insumos y proponer una estrategia de educación mediática e informacional para padres y profesores en este ámbito. Se dará cuenta de la primera etapa, cuantitativa, en la que padres y madres $(n=146)$ con hijos entre 8 y 13 años de edad respondieron un cuestionario autoadministrado sobre sus percepciones de la relación establecida por los niños con distintas pantallas y para explorar sus prácticas de mediación parental.

Somos ciudadanos mediáticos (Winocur, 2002), por lo que resulta relevante investigar la experiencia práctica y simbólica de las tecnologías en la vida cotidiana de distintos sectores socio-culturales (Winocur, 2007). En la medida en que los padres se empoderen, producto de la alfabetización en medios, podrán transmitir su experiencia y ser capaces de ponerse en el lugar de sus hijos, facilitando así un mejor ejercicio de la ciudadanía. Livingstone (2011) sugiere que la educación en medios va más allá de adquirir un conjunto de habilidades analíticas y comunicacionales, pues es una forma de transformar las audiencias y las sociedades. Investigaciones anteriores sobre niños y adolescentes ante múltiples pantallas realizadas en Chile, en particular las asociadas a la red internacional Generaciones Interactivas lideradas por Bringué-Sala y Sádaba-Chalezquer (2008) y por Bringué-Sala, Sádaba-Chalezquer y Tolsà-Caballero (2010), consideran la variable de la mediación educativa adulta y familiar en el uso de los medios y de las tecnologías, aunque dando la voz a los usuarios jóvenes, en distintos rangos de edad (entre 6 y 9 años y entre 10 y 18 años), utilizando dos cuestionarios diferentes. Por ese motivo nos parece relevante contribuir al mismo tema de investigación desde la perspectiva de los padres. 


\section{Estado de la cuestión: importancia de la mediación parental en la sociedad digital}

Desde la instalación de la televisión en los hogares, el medio rey del siglo XX se ha asociado a la parentalidad positiva que los adultos medien la relación que sus hijos establecen con los medios de comunicación (Aierbe; Medrano; Orejudo, 2008; Nathanson, 1999).

La educación en medios se asocia con la libertad de expresión e información, ya que permite a los ciudadanos comprender las funciones de los medios de comunicación y otros proveedores de información, evaluar críticamente su contenido y tomar decisiones informadas como usuarios y productores de información y contenido multimedia (Unesco, 2018). Sin embargo, al menos en Chile, la formación de padres y profesores en este ámbito no ha sido una práctica contemplada en las políticas públicas, en la formación universitaria o que esté presente en los currículos escolares (De-Fontcuberta; Guerrero, 2011). En un ecosistema comunicacional enriquecido, convergente y digital como el actual, caracterizado por la co-presencia de múltiples pantallas (Aguaded, 2011; Sádaba-Chalezquer; Bringué-Sala, 2010; Morduchowicz, 2008) y por nuevas formas de comunicar a través de las narrativas transmedia (Scolari, 2016) es aún más necesario.

Cada vez a más temprana edad los niños usan diversas tecnologías de información y comunicación, acceden a contenidos de muy distinta índole y calidad, toman contacto con otras personas que no necesariamente conocen y generan nuevas prácticas de comunicación en las que son protagonistas, como afirma Aguaded (2012), "de manera vicaria y directa" (p. 7). De ahí que

"uno de los retos actuales de las familias, las escuelas y la política educativa es incrementar los beneficios y evitar los riesgos del uso de internet en la infancia y la adolescencia" (Bartau-Rojas; Aierbe-Barandiaran; Oregui-González, 2018, p. 72).

Las estrategias de mediación parental revisten una importancia particular, en especial durante la infancia, una etapa crucial de desarrollo psicológico, afectivo, moral y cognitivo y que requiere de la guía de los adultos a su cargo. Ello importa porque como afirman Mihailidis y Viotty (2017), la educación en medios se mantiene periférica en el curriculum y en la clase, en un entorno en que es más necesaria que nunca, cuando se amplifica la sociedad del espectáculo y se multiplican las estrategias de creación de noticias falsas en las redes sociales y en los medios. Dudaceck (2015) considera una oportunidad poder aprovechar la narrativa transmedia en la educación de las nuevas generaciones.

A diferencia de antaño, los niños y los adolescentes acuden antes y primero a las pantallas que a los adultos con los que interactúan en la escuela o en el seno de la familia (Orozco; Franco, 2014). Según estos autores, el entorno digital modifica el rol tradicional de los padres y educadores, pues cambian

"las lógicas de intercambio informativo y formativo" (...) donde las nuevas generaciones acuden para aprender o conocer algo" (Orozco; Franco, 2014, p. 24).

En esa línea, algunos estudios han constatado los cambios acelerados del ecosistema comunicacional y la necesidad de establecer nuevas formas de implementación de estrategias de alfabetización transmedia que respondan al entorno de la sociedad digital (Ciastellardi; Di-Rosario, 2015; Costa, 2011; Condeza; De-Fontcuberta, 2015; Frau-Meiggs, 2012; Potter, 2010; Scolari, 2016). Según Orozco y Franco (2014), vivimos "al filo de las pantallas" y del lenguaje audiovisual, fenómeno que trastoca

"de múltiples formas y maneras los procesos de enseñanza/aprendizaje que, tradicionalmente, se habían volcado a la escuela y al libro como entidades unívocas del saber y el conocimiento" (Orozco; Franco, 2014, p. 22).

Ello requiere desarrollar la competencia mediática en la ciudadanía, y especialmente en los jóvenes, niños y niñas,

"para que puedan ejercer de forma crítica y activa su papel ante los medios... clave en esta sociedad de 'prosumidores mediáticos'” (García-Ruiz; Ramírez-García; Rodríguez-Rossell, 2014, p. 15).

Además, la sociedad de la información y el entorno digital provocan cambios en distintos ámbitos de nuestra vida cotidiana, como

"el modo de gobernar, los entornos de trabajo, la forma de acceder a la información, los modos de gestionar el conocimiento, la manera de comunicarnos y de relacionarnos, la forma de consumir, etc." (Santiago-Campión; Navaridas-Nalda; Andía-Celaya, 2016, p. 146).

Comunicar y educar en el mundo que viene (Aparici; García-Marín, 2017) se diferencia en gran medida de las primeras teorizaciones sobre la mediación parental, es decir las interesadas en el rol activo en la gestión y la regulación de los padres de las experiencias de los niños con la televisión a través de la comunicación interpersonal, como una forma de socialización (Schofield-Clarck, 2011). Esta autora sugiere que en el contexto digital, la mediación parental tiene el reto de pasar a una fase de aprendizaje participativo entre los padres e hijos. Ello se sumaría a las tres formas tradicionales en que, citando a Nathanson (1998), se ha definido y estudiado tradicionalmente la mediación de los padres en relación con los medios de comunicación, en particular la televisión:

- activa: por medio de la conversación sobre los contenidos vistos en televisión;

- restrictiva: por establecimiento de normas y de regulaciones;

- compartida: viendo televisión con sus hijos. 
Ferrés-Prats (2010) inscribe la educomunicación en una cultura participativa, la misma que según Bruns (2008) estará liderada en el futuro por produsers, productores-usuarios que crean, comparten y expanden contenidos en distintas plataformas y redes. Advierte que en la economía post-industrial las instituciones educativas requieren enseñar cuatro nuevas competencias centrales: creatividad, colaboración, crítica y comunicación. La necesidad de repensar constantemente la educación en medios en el contexto de la sociedad digital también es subrayada por Jenkins, Ito y Boyd (2015), quienes notan la emergencia de nuevas tensiones relacionadas con la participación de los más jóvenes en plataformas digitales y redes sociales de comunicación móvil, así como el uso comercial que se hace de sus datos personales en el contexto de la industria cultural.

Por esos motivos, en sociedades en las que hasta Robinson Crusoe tiene celular (Winocur, 2009) y considerando la necesidad de nuevos alfabetismos que desafían a los gobiernos a nivel mundial (Pereira; Pinto; Pereira, 2012), resulta relevante también abordar la necesidad de alfabetismo transmedia de los adultos, y en particular de los padres. Ciastellardi y Di-Rosario lo definen como

"un set de herramientas teóricas y analíticas para adquirir las habilidades que se requieren para comprender críticamente los caracteres y las posibilidades de la cultura emergente" (Ciastellardi; Di-Rosario, 2015, p. 12).

Según explica Koltay (2011), la alfabetización en medios es un concepto sombrilla, dinámico, debajo del cual se cobijan otras perspectivas, como la information literacy y la digital literacy. Independientemente de esta visión plural existe consenso en las competencias/dimensiones que engloba, a pesar de los cambios en el ecosistema de los medios: acceso, análisis, evaluación y producción de contenidos (Aufderheide, 1993; Buckingham, 2005; Koltay, 2011; Livingstone, 2004; 2011; Livingstone; Van-Couvering; Thumim, 2005; Scolari, 2016).

Scolari (2016) advierte que el concepto de alfabetización transmedia ha evolucionado en la práctica y en la teoría desde categorías tempranamente enunciadas como la de alfabetización en medios (Aufderheide, 1993). En el actual escenario algunos apuestan por hablar de multialfabetismo (Area-Moreira, 2010), alfabetización múltiple y multimodal (Koltay, 2011) o simplemente de alfabetización transmedia, un concepto defendido por Scolari (2016), quien ve en él la posibilidad de enriquecer la concepción tradicional de alfabetización mediática. Este tema ha tomado tal relevancia que ha sido incluido en el diseño de las políticas públicas en Europa (Buckingham, 2009; Comisión Europea, 2007; Livingstone, 2004).

En el proceso de alfabetización transmedia no sólo deben intervenir educadores y comunicadores, sino que resultan clave otros actores, como la familia (Chiavassa, 2015), especialmente los padres. Bien indicaba Castells que

"la educación es el recurso clave en un mundo en el que la fuente de poder y riqueza es la capacidad de procesar información para transformarla en conocimiento aplicado" (Castells, 2007, párr. 3).

En esta misma línea de pensamiento, la alfabetización transmedia contribuye al ejercicio de la democracia, la participación y la ciudadanía activa, también a la economía del conocimiento, la competitividad y la elección; de la misma forma que es una expresión cultural y de realización personal (Livingstone; Van-Couvering; Thumim, 2005).

La pregunta general que guía esta investigación es la siguiente: ¿Cuáles son las prácticas de mediación que ejercen los padres sobre el uso que hacen sus hijos de las TIC y cuáles son las percepciones que las sustentan? En cuanto a los objetivos específicos, se buscó:

1. Identificar el perfil tecnológico de los padres, relacionarlo con sus prácticas de mediación parental y con las percepciones que tienen sobre el uso que hacen sus hijos de las TIC;

2. Explorar las prácticas de mediación parental sobre el uso que hacen sus hijos de las TIC;

3. Analizar las percepciones que tienen los padres sobre el uso que hacen sus hijos de las TIC.

\section{Metodología}

En la investigación se empleó el enfoque sistémico de Jordan (2004), referido por Padilla-Walker y Coyne (2011). Como afirman las autoras,

"la experiencia de los niños y niñas con los medios se inscribe en un conjunto de interacciones mucho más complejas de comunicación, con los padres, madres, profesores y otras personas, que tienen que ser examinadas. Es desde este contexto más amplio que se debe entender el efecto del uso de los medios" (Padilla-Walker; Coyne, 2011, p. 706).

Las percepciones y referencias a prácticas de mediación de los padres de niños de entre 8 y 13 años de edad se abordaron en dos fases. Una primera, cuantitativa, la difundida en este trabajo, consistió en crear y validar un cuestionario auto-administrado de 52 items, con un total de 18 preguntas de matriz simple, 18 preguntas con respuesta única, 9 pre- 
guntas de respuesta múltiple, 6 preguntas abiertas numéricas o de una palabra y 1 de matriz simple abierta numérica. Tras la validación del cuestionario se determinó una muestra por conveniencia, a partir de los datos estadísticos sobre los establecimientos docentes de zona urbana de la Región Metropolitana de Santiago de Chile publicados por el Centro de Estudios del Ministerio de Educación de Chile (Mineduc, 2014). Se consideraron los tres tipos de dependencia para la distribución representativa de la realidad chilena:

- pública-municipal: 29 centros;

- subvencionada: 88 ;

- privada: 34.

El cuestionario se aplicó, previo consentimiento informado, entre octubre y noviembre de 2016.

Las variables utilizadas para explorar el perfil tecnológico de los padres, sus percepciones sobre el uso que hacen sus hijos de las TIC y las prácticas de mediación que asumen en este contexto fueron las siguientes:

Variables sobre el perfil tecnológico de los padres:

- Uso de internet, medido en horas: pregunta 14, de respuesta única.

- Índice de cantidad de actividades que realiza online en el último mes: Buscar información en internet, chatear, enviar correos electrónicos, uso de redes sociales (Facebok, Twitter), usar Whatsapp con padres, usar Whatsapp con profesores, leer noticias o artículos de internet, usar juegos en internet: pregunta 17, matriz simple.

Variables sobre las percepciones de los padres con respecto a la influencia de la tecnología en:

- Habilidades académicas de los hijos: desarrollo de habilidades académicas como buscar, analizar, seleccionar y generar información: pregunta 21, matriz simple.

- Desarrollo y desempeño de los hijos: desarrollo personal, esparcimiento y recreación, relaciones sociales, desempeño tanto escolar como en educación superior y en el futuro entorno laboral: pregunta 20, matriz simple.

- Ejercicio de derechos de los hijos: derecho a informarse, a expresar su opinión, a educarse y aprender, a asociarse y a manifestar la identidad personal: pregunta 22, matriz simple.

- Comunicación de los hijos con otros: comunicación con sus padres, con personas menores que ellos, de igual edad y mayores: pregunta 23, matriz simple.

Variables de supervisión y acompañamiento parental en el uso de internet:

- Índice de comprobación de uso de internet: sitios web, mensajes en e-mail o cuenta de mensajería instantánea con y sin la presencia de su hijo, perfil de red social o comunidad online con y sin la presencia de su hijo, qué amigos o contactos añade a su perfil de red social o cuenta de mensajería instantánea/Messenger con y sin la presencia de su hijo: pregunta 39 , matriz simple.

- Índice de uso de aplicaciones y programas de comprobación de bloqueo que como padres consideran inadecuados: bloqueos de sitios web, seguimiento de sitios web visitados, bloqueo de algunos canales de TV, servicio o contrato que limite el tiempo que el hijo pase en internet, servicio o contrato que limite el tiempo que el hijo pase viendo TV, software/aplicación para prevenir spam o virus: pregunta 40, pregunta matriz simple. Premio y/o castigo para distracción de su hijo mientras padres se ocupan de otros asuntos, cuando le pide ayuda o le enseñe a usar tecnología, necesita que su hijo se calme, no interrumpa a los adultos, coma su comida o duerma, su hijo está aburrido, su hijo le solicita ayuda con alguna tarea del colegio: pregunta 45, matriz simple.

\section{Resultados}

\subsection{Descripción de la muestra}

Del total de casos válidos de la muestra $(n=146)$, un $81,7 \%(n=121)$ son mujeres y el $17,1 \%(n=25)$ son hombres. El rango de edad se concentra entre los 33 y 42 años, con un $45,9 \%(n=67)$, seguido por un $27,4 \%$ ( $n=40$ ) entre los 43 años o más y $26 \%, 7(n=39)$ entre los 23 y 32 años.

El 98,6\% ( $n=144)$ de las personas que responden la encuesta son madres o padres de los estudiantes. Del total de las madres, un $51,2 \%(n=62)$ tiene educación superior completa o incompleta, un $42,1 \%(n=51)$ educación media completa o incompleta y un $6,6 \%(n=8)$ educación básica completa o incompleta. Por su parte, un $60 \%(n=15)$ de los padres tiene educación superior completa o incompleta, un $36 \%(n=9)$ educación media completa o incompleta y un $4 \%(n=1)$ educación básica completa o incompleta.

Cada objetivo de la investigación se aborda en los siguientes apartados.

\subsection{Perfil de usuario tecnológico de los padres}

Tal como se observa en la tabla 1, un $46 \%$ de los padres de la muestra realiza entre 5 y 6 actividades. La proporción entre aquellos que tienen entre 43 años o más es significativamente menor que los otros dos grupos de edades. 
Tabla 1. Cantidad de actividades en internet en el último mes según edad de los padres

\begin{tabular}{|c|c|c|c|c|c|}
\hline \multirow{2}{*}{\multicolumn{2}{|c|}{ N. actividades en internet }} & \multicolumn{3}{|c|}{ Edad de los padres } & \multirow{3}{*}{$\begin{array}{c}\text { Total } \\
3\end{array}$} \\
\hline & & \multirow{2}{*}{$\frac{23 \text { a } 32 \text { años }}{1}$} & \multirow{2}{*}{$\frac{33 \text { a } 42 \text { años }}{1}$} & \multirow{2}{*}{43 años o más } & \\
\hline Nlingunz actividad & $\mathrm{n}$ & & & & \\
\hline Tinguna actividad & $\%$ & $2,6 \%$ & $1,6 \%$ & $2,6 \%$ & $2,2 \%$ \\
\hline \multirow{2}{*}{1 a 2 actividades } & $\mathrm{n}$ & 6 & 7 & 6 & 19 \\
\hline & $\%$ & $15,4 \%$ & $11,3 \%$ & $15,8 \%$ & $13,7 \%$ \\
\hline \multirow{2}{*}{3 a 4 actividades } & $\mathrm{n}$ & 8 & 8 & 9 & 25 \\
\hline & $\%$ & $20,5 \%$ & $12,9 \%$ & $23,7 \%$ & $18,0 \%$ \\
\hline \multirow{2}{*}{5 a 6 actividades } & $\mathrm{n}$ & 18 & 32 & 14 & 64 \\
\hline & $\%$ & $46,2 \%$ & $51,6 \%$ & $36,8 \%$ & $46,0 \%$ \\
\hline \multirow{2}{*}{7 a 8 actividades } & $\mathrm{n}$ & 6 & 14 & 8 & 28 \\
\hline & $\%$ & $15,4 \%$ & $22,6 \%$ & $21,1 \%$ & $20,1 \%$ \\
\hline \multirow{2}{*}{ Total } & $n$ & 39 & 62 & 38 & 139 \\
\hline & $\%$ & $100 \%$ & $100 \%$ & $100 \%$ & $100 \%$ \\
\hline
\end{tabular}

$\chi^{2}=4,089 ; p=0,849$

Por otro lado, como se observa en la tabla 2, la cantidad promedio de uso de internet dependerá de la edad de los padres. Quienes dedican menos horas, entre 0 a 1 horas, son los de mayor edad y aquellos que le dedican entre 1 a 3 son los más jóvenes.

Tabla 2. Horas promedio de uso de internet según edad de los padres

\begin{tabular}{|c|c|c|c|c|c|}
\hline \multirow{2}{*}{\multicolumn{2}{|c|}{ Horas uso internet }} & \multicolumn{3}{|c|}{ Edad de los padres } & \multirow{3}{*}{$\begin{array}{c}\text { Total } \\
25\end{array}$} \\
\hline & & \multirow{2}{*}{$\frac{23 \text { a } 32 \text { años }}{5}$} & \multirow{2}{*}{$\begin{array}{c}23 \text { a } 32 \text { años } \\
9\end{array}$} & \multirow{2}{*}{$\frac{\mathbf{2 3} \text { a } \mathbf{3 2} \text { años }}{11}$} & \\
\hline 0.1 hor & $\mathrm{n}$ & & & & \\
\hline a tillua & $\%$ & $14,3 \%$ & $14,1 \%$ & $28,9 \%$ & $18,2 \%$ \\
\hline \multirow{2}{*}{1 a 3 horas } & $n$ & 13 & 31 & 10 & 54 \\
\hline & $\%$ & $37,1 \%$ & $48,4 \%$ & $26,3 \%$ & $39,4 \%$ \\
\hline \multirow{2}{*}{3 a 6 horas } & $\mathrm{n}$ & 13 & 9 & 6 & 28 \\
\hline & $\%$ & $37,1 \%$ & $14,1 \%$ & $15,8 \%$ & $20,4 \%$ \\
\hline \multirow{2}{*}{6 a 8 horas } & $\mathrm{n}$ & 2 & 7 & 7 & 16 \\
\hline & $\%$ & $5,7 \%$ & $10,9 \%$ & $18,4 \%$ & $11,7 \%$ \\
\hline \multirow{2}{*}{8 o más horas } & $\mathrm{n}$ & 2 & 8 & 3 & 13 \\
\hline & $\%$ & $5,7 \%$ & $12,5 \%$ & $7,9 \%$ & $9,5 \%$ \\
\hline \multirow{2}{*}{ Ninguna } & $\mathrm{n}$ & 0 & 0 & 1 & 1 \\
\hline & $\%$ & $0,0 \%$ & $0,0 \%$ & $2,6 \%$ &, $7 \%$ \\
\hline \multirow{2}{*}{ Total } & $\mathrm{n}$ & 35 & 64 & 38 & 137 \\
\hline & $\%$ & $100 \%$ & $100 \%$ & $100 \%$ & $100 \%$ \\
\hline
\end{tabular}

$\chi^{2}=19,194 ; p=0,038$

El perfil tecnológico de los padres fue construido como un índice compuesto por las dos variables descritas anteriormente:

- realizar actividades en internet en el último mes;

- cantidad de horas promedio de uso de internet.

Según este análisis, no existen diferencias entre el perfil de usuario y la edad de los apoderados. La mayoría de ellos están muy relacionados con las TIC. Tampoco influye en el perfil de usuario de TIC de los padres el tipo de centro en el que está matriculado su hijo. Podría pensarse que los colegios municipales, que por lo general tienen alumnos de familias de menor ingreso económico, contarían con padres menos tecnológicos que los privados, pero no ocurre así. Lo que sí determina el perfil de usuario de los padres es su nivel de escolaridad. Los padres que han llegado a la educación superior tienen una relación notablemente mayor con las TIC que aquellos que sólo tuvieron acceso a una educación básica.

\subsection{Prácticas parentales de mediación en el uso de TIC}

Una alta proporción de los encuestados, independientemente de la edad, declara comprobar lo que su hijo hace en internet (entre $87,5 \%$ y $91,4 \%$ ). Para ello emplean programas y aplicaciones para bloquear contenidos que consideran inadecuados. Ambas prácticas son más empleadas por los padres de menor edad en la muestra, tal como se observa en la tabla 3, aquellos que podemos identificar como nativos digitales. 
Tabla 3. Comprobación de uso de internet y uso de programas/aplicaciones de computación para evitar contenidos inadecuados según edad de los padres

\begin{tabular}{|c|c|c|c|c|c|c|c|c|c|}
\hline & & \multirow{2}{*}{\multicolumn{4}{|c|}{$\begin{array}{c}\text { Comprobación de uso de internet } \\
\text { Edad de los padres }\end{array}$}} & \multicolumn{4}{|c|}{$\begin{array}{c}\text { Uso de programas o aplicaciones de computación } \\
\text { para evitar contenidos inadecuados }\end{array}$} \\
\hline \multirow{2}{*}{\multicolumn{2}{|c|}{ Comprobación }} & & & & & & de los pa & & \\
\hline & & $\begin{array}{l}23 \text { a } 32 \\
\text { años }\end{array}$ & $\begin{array}{l}33 \text { a } 42 \\
\text { años }\end{array}$ & $\begin{array}{l}43 \text { años o } \\
\text { más }\end{array}$ & Total & $\begin{array}{c}23 \text { a } 32 \\
\text { años }\end{array}$ & $\begin{array}{c}33 \text { a } 42 \\
\text { años }\end{array}$ & $\begin{array}{l}43 \text { años } 0 \\
\text { más }\end{array}$ & Total \\
\hline \multirow{2}{*}{ No } & $\mathrm{n}$ & 3 & 6 & 4 & 13 & 13 & 25 & 15 & 53 \\
\hline & $\%$ & $8,6 \%$ & $10,2 \%$ & $12,5 \%$ & $10,3 \%$ & $33,3 \%$ & $37,3 \%$ & $37,5 \%$ & $36,3 \%$ \\
\hline \multirow{2}{*}{ Sí } & $\mathrm{n}$ & 32 & 53 & 28 & 113 & 26 & 42 & 25 & 93 \\
\hline & $\%$ & $91,4 \%$ & $89,8 \%$ & $87,5 \%$ & $89,7 \%$ & $66,7 \%$ & $62,7 \%$ & $62,5 \%$ & $63,7 \%$ \\
\hline \multirow{2}{*}{ Total } & $\mathrm{n}$ & 35 & 59 & 32 & 126 & 39 & 67 & 40 & 146 \\
\hline & $\%$ & $100 \%$ & $100 \%$ & $100 \%$ & $100 \%$ & $100 \%$ & $100 \%$ & $100 \%$ & $100 \%$ \\
\hline
\end{tabular}

Otra de las prácticas parentales recurrentes en el contexto chileno es utilizar la tecnología como desincentivo o premio a conductas de sus hijos. Un 41,2\% de los padres de la muestra afirman haberla utilizado alguna vez para premiar 0 desincentivar alguna conducta que consideran inadecuada. Aquellos con mayor edad recurren menos a esta práctica. Las respuestas en este caso podrían estar sesgadas, si se piensa en el posible efecto de espiral del silencio en este ítem. Recurrir a la tecnología como castigo-recompensa, podría considerarse socialmente como una práctica poco adecuada.

Tabla 4. Uso de tecnología como premio o desincentivo a conductas inadecuadas según edad de los padres

\begin{tabular}{|l|c|c|c|}
\hline \multirow{2}{*}{ Frecuencia } & \multicolumn{3}{|c|}{ Edad de los padres } \\
\cline { 2 - 4 } & $\mathbf{2 3}$ a 32 años & $\mathbf{3 3}$ a 42 años & $\mathbf{4 3}$ años o más \\
\hline \multirow{2}{*}{ Me ha ocurrido muchas veces } & 9 & 13 & 6 \\
\hline \multirow{2}{*}{ Me ha ocurrido alguna vez } & $24,3 \%$ & $22,8 \%$ & $16,2 \%$ \\
\hline \multirow{2}{*}{ Nunca me ha ocurrido } & 17 & 23 & 14 \\
\cline { 2 - 5 } & $45,9 \%$ & $40,4 \%$ & $37,8 \%$ \\
\hline \multirow{2}{*}{ Total } & 11 & 21 & 17 \\
\cline { 2 - 5 } & $29,7 \%$ & $36,8 \%$ & $45,9 \%$ \\
\hline
\end{tabular}

$\chi^{2}=2,289 ; p=0,038$

Asimismo, es reiterada la posición de los padres de facilitar el uso de TIC a sus hijos en situaciones en las que necesitan tiempo para ellos mismos y no pueden encargarse de entretener a los menores. El $57 \%$ de los encuestados admite haber facilitado el uso de TIC a sus hijos cuando necesitaba mantenerlo distraído mientras se ocupaba de asuntos de adultos. También más de la mitad (52\%) reconoce que ha facilitado el uso de TIC a sus hijos para que estos le enseñen a usar las nuevas tecnologías. Aunque en menor medida $(15,4 \%)$, un porcentaje de padres recurre al uso de las tecnologías ante la necesidad de que los hijos se calmen, no interrumpan a los adultos, coman su comida o se duerman. En esta situación también puede ocurrir lo que describíamos anteriormente: la cifra podría ser mayor debido a que los convencionalismos sociales a veces conllevan a las personas a responder según el deber ser y no lo que en realidad es.

Una de las prácticas parentales recurrentes en el contexto chileno es utilizar la tecnología como desincentivo o premio a conductas de sus hijos, lo cual podría considerarse socialmente como poco adecuado al contestar la encuesta

Al observar lo anterior por rango de edad, los adultos más jóvenes son quienes más facilitan las TIC a sus hijos mientras ellos se ocupan de otros asuntos. En cambio, los padres de más edad son los que en mayor medida ofrecen el uso de internet a sus hijos para que estos les enseñen a usar la tecnología, pues tienen menos habilidades que los más jóvenes, considerados nativos digitales (tabla 5).

Los padres que respondieron los cuestionarios proporcionan el uso de TIC a sus hijos cuando estos se encuentran aburridos o deben hacer una tarea, observándose diferencia según la edad de los padres:

- los más jóvenes son más propensos a facilitar a sus hijos el uso de las TIC si el menor está aburrido, a diferencia de la postura que asumen los de mayor edad en este sentido, quienes prefieren hacerlo en menor medida;

- el $100 \%$ de los padres de mayor edad facilitan el uso de la tecnología a sus hijos para realizar tareas escolares, a diferencia de los más jóvenes (tabla 6). 
Tabla 5. Facilitación de uso de tecnología a su hijo/a cuando: necesita distracción mientras se ocupa de otros asuntos, usted le pide que le ayude o le enseñe a usar la tecnología y necesita que se calme, no interrumpa a los adultos, coma su comida o se duerma, según edad de los padres

\begin{tabular}{|c|c|c|c|c|c|c|c|c|c|c|c|c|}
\hline \multirow{3}{*}{$\begin{array}{l}\text { Facilita- } \\
\text { ción uso }\end{array}$} & \multicolumn{4}{|c|}{$\begin{array}{l}\text { Necesita distracción mientras usted se } \\
\text { ocupa de otros asuntos }\end{array}$} & \multicolumn{4}{|c|}{$\begin{array}{c}\text { Usted le pide que le ayude o le enseñe } \\
\text { a usar las tecnologías }\end{array}$} & \multicolumn{4}{|c|}{$\begin{array}{l}\text { Necesita que se calme, no interrumpa } \\
\text { a los adultos, coma su comida o se } \\
\text { duerma }\end{array}$} \\
\hline & \multicolumn{3}{|c|}{ Edad de los padres } & \multirow[b]{2}{*}{ Total } & \multicolumn{3}{|c|}{ Edad de los padres } & \multirow[b]{2}{*}{ Total } & \multicolumn{3}{|c|}{ Edad de los padres } & \multirow[b]{2}{*}{ Total } \\
\hline & $\begin{array}{l}23 \text { a } 32 \\
\text { años }\end{array}$ & $\begin{array}{l}33 \text { a } 42 \\
\text { años }\end{array}$ & $\begin{array}{l}43 \text { años } \\
\text { o más }\end{array}$ & & $\begin{array}{l}23 \text { a } 32 \\
\text { años }\end{array}$ & $\begin{array}{l}33 \text { a } 42 \\
\text { años }\end{array}$ & $\begin{array}{l}43 \text { años } \\
\text { o más }\end{array}$ & & $\begin{array}{l}23 \text { a } 32 \\
\text { años }\end{array}$ & $\begin{array}{l}33 \text { a } 42 \\
\text { años }\end{array}$ & $\begin{array}{l}43 \text { años } \\
\text { o más }\end{array}$ & \\
\hline \multirow{2}{*}{ No } & 17 & 32 & 23 & 72 & 29 & 29 & 19 & 77 & 29 & 47 & 28 & 104 \\
\hline & $48,6 \%$ & $55,2 \%$ & $63,9 \%$ & $55,8 \%$ & $78,4 \%$ & $51,8 \%$ & $52,8 \%$ & $59,7 \%$ & $82,9 \%$ & $88,7 \%$ & $80,0 \%$ & $84,6 \%$ \\
\hline \multirow{2}{*}{ Sí } & 18 & 26 & 13 & 57 & 8 & 27 & 17 & 52 & 6 & 6 & 7 & 19 \\
\hline & $51,4 \%$ & $44,8 \%$ & $36,1 \%$ & $44,2 \%$ & $21,6 \%$ & $48,2 \%$ & $47,2 \%$ & $40,3 \%$ & $17,1 \%$ & $11,3 \%$ & $20,0 \%$ & $15,4 \%$ \\
\hline \multirow{2}{*}{ Total } & 35 & 58 & 36 & 129 & 37 & 56 & 36 & 129 & 35 & 53 & 35 & 123 \\
\hline & $100 \%$ & $100 \%$ & $100 \%$ & $100 \%$ & $100 \%$ & $100 \%$ & $100 \%$ & $100 \%$ & $100 \%$ & $100 \%$ & $100 \%$ & $100 \%$ \\
\hline
\end{tabular}

Tabla 6. Facilitación de uso de tecnología a su hijo/a cuando está aburrido y cuando le solicita ayuda con alguna tarea para el colegio, según edad de los padres

\begin{tabular}{|c|c|c|c|c|c|c|c|c|c|}
\hline \multirow{3}{*}{\multicolumn{2}{|c|}{ Facilitación Uso }} & \multicolumn{4}{|c|}{ Está aburrido } & \multicolumn{4}{|c|}{ Le solicita ayuda con alguna tarea para el colegio } \\
\hline & & \multicolumn{3}{|c|}{ Edad de los padres } & \multirow{3}{*}{$\begin{array}{c}\text { Total } \\
62 \\
\end{array}$} & \multicolumn{3}{|c|}{ Edad de los padres } & \multirow{3}{*}{$\begin{array}{c}\text { Total } \\
6\end{array}$} \\
\hline & & $\begin{array}{c}23 \text { a } 32 \\
\text { años }\end{array}$ & $\begin{array}{c}33 \text { a } 42 \\
\text { años }\end{array}$ & $\begin{array}{c}43 \text { años o } \\
\text { más }\end{array}$ & & $\begin{array}{c}23 \text { a } 32 \\
\text { años }\end{array}$ & $\begin{array}{c}33 \text { a } 42 \\
\text { años }\end{array}$ & $\begin{array}{l}43 \text { años o } \\
\text { más }\end{array}$ & \\
\hline \multirow{2}{*}{ No } & $\mathrm{n}$ & 16 & 26 & 20 & & 3 & 3 & 0 & \\
\hline & $\%$ & $44,4 \%$ & $45,6 \%$ & $54,1 \%$ & $47,7 \%$ & $7,7 \%$ & $4,8 \%$ & $0,0 \%$ & $4,3 \%$ \\
\hline \multirow{2}{*}{ Sí } & $n$ & 20 & 31 & 17 & 68 & 36 & 59 & 37 & 132 \\
\hline & $\%$ & $55,6 \%$ & $54,4 \%$ & $45,9 \%$ & $52,3 \%$ & $92,3 \%$ & $95,2 \%$ & $100 \%$ & $95,7 \%$ \\
\hline \multirow{2}{*}{ Total } & $\mathrm{n}$ & 36 & 57 & 37 & 130 & 39 & 62 & 37 & 138 \\
\hline & $\%$ & $100 \%$ & $100 \%$ & $100 \%$ & $100 \%$ & $100 \%$ & $100 \%$ & $100 \%$ & $100 \%$ \\
\hline
\end{tabular}

\subsection{Percepciones de los padres sobre el uso que hacen sus hijos de las TIC}

Las prácticas parentales descritas anteriormente están sustentadas en varias creencias y acciones que demanda la vida cotidiana moderna. Son relevantes las percepciones que se tienen sobre el uso de las TIC en varios ámbitos de la infancia: influencia en:

- adquisición de habilidades académicas de los niños;

- su futuro estudiantil, profesional y laboral;

- ejercicio de sus derechos;

- relaciones sociales que establece;

- la comunicación que establecen con sus padres.

Por estas razones, en la segunda parte del estudio exploramos también las percepciones de los padres sobre el uso que hacen sus hijos de las TIC.

Considerando que la alfabetización en medios contempla las siguientes competencias/ dimensiones de acceso, análisis, evaluación y producción de contenidos (Aufderheide, 1993; Buckingham, 2005; Koltay, 2011; Livingstone, 2004, 2011; Livingstone; Van-Couvering; Thumim, 2005; Scolari, 2016), se observa que los padres en relación con las dimensiones anteriores perciben que internet tiene una influencia positiva, sobre el $90 \%$, para aquellas habilidades relacionadas con el acceso y el análisis (tabla 7). No se identifican diferencias por edad de los padres en la percepción de influencia de internet en el acceso a contenidos, pero sí en su análisis. Un mayor número de padres de menor edad creen que el uso de internet no influye en el análisis que puedan desarrollar sus hijos sobre determinado tema, mientras que los de mayor edad creen que influye positivamente.
Un mayor número de padres de menor edad creen que el uso de internet no influye en el análisis que puedan desarrollar sus hijos sobre determinado tema, mientras que los de mayor edad creen que influye positivamente 
Tabla 7. Influencia de internet en la adquisición de la habilidad académica de su hijo para: buscar información (acceso a contenidos) y analizar información (análisis de contenidos), según edad de los padres

\begin{tabular}{|c|c|c|c|c|c|c|c|c|c|}
\hline & & \multicolumn{4}{|c|}{ Buscar información (acceso) } & \multicolumn{4}{|c|}{ Analizar información (análisis) } \\
\hline \multirow{2}{*}{\multicolumn{2}{|c|}{ Tipo de influencia }} & \multicolumn{3}{|c|}{ Edad de los padres } & \multirow[b]{2}{*}{ Total } & \multicolumn{3}{|c|}{ Edad de los padres } & \multirow[b]{2}{*}{ Total } \\
\hline & & $\begin{array}{c}23 \text { a } 32 \\
\text { años }\end{array}$ & $\begin{array}{c}33 \text { a } 42 \\
\text { años }\end{array}$ & $\begin{array}{l}43 \text { años o } \\
\text { más }\end{array}$ & & $\begin{array}{c}23 \text { a } 32 \\
\text { años }\end{array}$ & $\begin{array}{c}33 \text { a } 42 \\
\text { años }\end{array}$ & $\begin{array}{l}43 \text { años o } \\
\text { más }\end{array}$ & \\
\hline \multirow{2}{*}{ Positivamente } & $\mathrm{n}$ & 34 & 54 & 30 & 118 & 18 & 40 & 25 & 46 \\
\hline & $\%$ & $91,9 \%$ & $94,7 \%$ & $96,8 \%$ & $94,4 \%$ & $56,3 \%$ & $83,3 \%$ & $78,1 \%$ & $93,9 \%$ \\
\hline \multirow{2}{*}{ No influye } & $n$ & 3 & 3 & 1 & 7 & 12 & 7 & 6 & 3 \\
\hline & $\%$ & $8,1 \%$ & $5,3 \%$ & $3,2 \%$ & $5,6 \%$ & $37,5 \%$ & $14,6 \%$ & $18,8 \%$ & $6,1 \%$ \\
\hline \multirow{2}{*}{ Negativamente } & $\mathrm{n}$ & 0 & 0 & 0 & 0 & 2 & 1 & 1 & 0 \\
\hline & $\%$ & $0 \%$ & $0 \%$ & $0 \%$ & $0 \%$ & $6,3 \%$ & $2,1 \%$ & $3,1 \%$ & $0,0 \%$ \\
\hline \multirow{2}{*}{ Total } & $\mathrm{n}$ & 37 & 57 & 31 & 125 & 32 & 48 & 32 & 49 \\
\hline & $\%$ & $100 \%$ & $100 \%$ & $100 \%$ & $100 \%$ & $100 \%$ & $100 \%$ & $100 \%$ & $100 \%$ \\
\hline
\end{tabular}

Por otro lado, en relación con la influencia de internet en la adquisición de las habilidades académicas de evaluación y producción de contenidos se observa una percepción de influencia positiva, aunque menor, a la de las dos habilidades académicas mencionadas anteriormente (tabla 8). Se observa que quienes tienen entre 33 y 42 años perciben en mayor proporción que la influencia es positiva para la habilidad de "selección de información", mientras que para la habilidad académica de "generar nueva información" es mayor la proporción para los más jóvenes de la muestra.

Tabla 8. Influencia en adquisición de la habilidad académica de su hijo de: seleccionar información (evaluación de contenidos) y para generar nueva información (producción de contenidos), según edad de los padres

\begin{tabular}{|c|c|c|c|c|c|c|c|c|c|}
\hline & & \multicolumn{4}{|c|}{ Seleccionar información (evaluación) } & \multicolumn{4}{|c|}{ Generar nueva información (producción) } \\
\hline \multirow{2}{*}{\multicolumn{2}{|c|}{ Tipo de influencia }} & \multicolumn{3}{|c|}{ Edad de los padres } & \multirow[b]{2}{*}{ Total } & \multicolumn{3}{|c|}{ Edad de los padres } & \multirow[b]{2}{*}{ Total } \\
\hline & & 23 a 32 & 33 a 42 & 43 años 0 & & 23 a 32 & 33 a 42 & 43 años o & \\
\hline \multirow{2}{*}{ Positivamente } & $\mathrm{n}$ & 25 & 46 & 21 & 92 & 28 & 40 & 19 & 87 \\
\hline & $\%$ & $78,1 \%$ & $93,9 \%$ & $77,8 \%$ & $85,2 \%$ & $84,8 \%$ & $81,6 \%$ & $70,4 \%$ & $79,8 \%$ \\
\hline \multirow{2}{*}{ No influye } & $\mathrm{n}$ & 6 & 3 & 4 & 13 & 5 & 9 & 8 & 22 \\
\hline & $\%$ & $18,8 \%$ & $6,1 \%$ & $14,8 \%$ & $12,0 \%$ & $15,2 \%$ & $18,4 \%$ & $29,6 \%$ & $20,2 \%$ \\
\hline \multirow{2}{*}{ Negativamente } & $\mathrm{n}$ & 1 & 0 & 2 & 3 & 0 & 0 & 0 & 0 \\
\hline & $\%$ & $3,1 \%$ & $0,0 \%$ & $7,4 \%$ & $2,8 \%$ & $0 \%$ & $0 \%$ & $0 \%$ & $0 \%$ \\
\hline \multirow{2}{*}{ Total } & $\mathrm{n}$ & 32 & 49 & 27 & 108 & 33 & 49 & 27 & 109 \\
\hline & $\%$ & $100,0 \%$ & $100,0 \%$ & $100,0 \%$ & $100,0 \%$ & $100 \%$ & $100 \%$ & $100 \%$ & $100 \%$ \\
\hline
\end{tabular}

El mayor porcentaje de los encuestados coincide en que internet influye positivamente en el esparcimiento y recreación de su hijo, así como en el desempeño que los menores tendrán en la educación superior. En relación con el esparcimiento y los diferentes tipos de desempeño (tablas 9 y 10), los padres perciben una influencia positiva de la tecnología, destacándose una mejor valoración para los dos grupos de mayor edad en la muestra.

Tabla 9. Influencia del uso de tecnología en el esparcimiento y desempeño en educación superior de su hijo según edad de los padres

\begin{tabular}{|c|c|c|c|c|c|c|c|c|c|}
\hline & & \multirow{2}{*}{\multicolumn{4}{|c|}{ Esparcimiento y recreación de su hijo }} & & & & \\
\hline & & & & & & \multicolumn{4}{|c|}{ Desempeño educación superior de su hijo } \\
\hline \multirow{2}{*}{\multicolumn{2}{|c|}{ Tipo de influencia }} & \multicolumn{3}{|c|}{ Edad de los padres } & \multirow{3}{*}{$\begin{array}{c}\text { Total } \\
52\end{array}$} & \multicolumn{3}{|c|}{ Edad de los padres } & \multirow{3}{*}{$\begin{array}{c}\text { Total } \\
77\end{array}$} \\
\hline & & \multirow{2}{*}{$\begin{array}{c}23 \text { a } 32 \\
\text { años } \\
12\end{array}$} & \multirow{2}{*}{$\begin{array}{c}33 \text { a } 42 \\
\text { años }\end{array}$} & \multirow{2}{*}{$\begin{array}{c}43 \text { años o } \\
\text { más } \\
18\end{array}$} & & \multirow{2}{*}{$\begin{array}{c}23 \text { a } 32 \\
\text { años } \\
20\end{array}$} & \multirow{2}{*}{$\begin{array}{c}33 \text { a } 42 \\
\text { años } \\
35\end{array}$} & \multirow{2}{*}{$\begin{array}{c}43 \text { años o } \\
\text { más } \\
22\end{array}$} & \\
\hline Dositivomonto & $\mathrm{n}$ & & & & & & & & \\
\hline Positivamente & $\%$ & $34,3 \%$ & $39,3 \%$ & $52,9 \%$ & $41,6 \%$ & $58,8 \%$ & $64,8 \%$ & $73,3 \%$ & $65,3 \%$ \\
\hline \multirow{2}{*}{ No influye } & $\mathrm{n}$ & 15 & 15 & 11 & 41 & 12 & 16 & 5 & 33 \\
\hline & $\%$ & $42,9 \%$ & $26,8 \%$ & $32,4 \%$ & $32,8 \%$ & $35,3 \%$ & $29,6 \%$ & $16,7 \%$ & $28,0 \%$ \\
\hline \multirow{2}{*}{ Negativamente } & $\mathrm{n}$ & 8 & 19 & 5 & 32 & 2 & 3 & 3 & 8 \\
\hline & $\%$ & $22,9 \%$ & $33,9 \%$ & $14,7 \%$ & $25,6 \%$ & $5,9 \%$ & $5,6 \%$ & $10,0 \%$ & $6,8 \%$ \\
\hline \multirow{2}{*}{ Total } & $\mathrm{n}$ & 35 & 56 & 34 & 125 & 34 & 54 & 30 & 118 \\
\hline & $\%$ & $100 \%$ & $100 \%$ & $100 \%$ & $100 \%$ & $100 \%$ & $100 \%$ & $100 \%$ & $100 \%$ \\
\hline
\end{tabular}


Tabla 10. Influencia de uso de tecnología en el desempeño escolar y laboral de su hijo según edad de los padres

\begin{tabular}{|c|c|c|c|c|c|c|c|c|c|}
\hline & & \multicolumn{4}{|c|}{ Desempeño escolar de su hijo } & \multicolumn{4}{|c|}{ Desempeño laboral de su hijo } \\
\hline \multirow{2}{*}{\multicolumn{2}{|c|}{ Tipo de influencia }} & \multicolumn{3}{|c|}{ Edad de los padres } & \multirow{3}{*}{$\begin{array}{c}\text { Total } \\
81\end{array}$} & \multicolumn{3}{|c|}{ Edad de los padres } & \multirow{3}{*}{$\begin{array}{r}\text { Total } \\
86\end{array}$} \\
\hline & & 23 a 32 & 33 a 42 & 43 años o & & 23 a 32 & 33 a 42 & 43 años o & \\
\hline \multirow{2}{*}{ Positivamente } & $\mathrm{n}$ & 20 & 37 & 24 & & 21 & 41 & 24 & \\
\hline & $\%$ & $55,6 \%$ & $59,7 \%$ & $63,2 \%$ & $59,6 \%$ & $60,0 \%$ & $74,5 \%$ & $72,7 \%$ & $69,9 \%$ \\
\hline \multirow{2}{*}{ No influye } & $\mathrm{n}$ & 11 & 14 & 6 & 31 & 13 & 12 & 9 & 34 \\
\hline & $\%$ & $30,6 \%$ & $22,6 \%$ & $15,8 \%$ & $22,8 \%$ & $37,1 \%$ & $21,8 \%$ & $27,3 \%$ & $27,6 \%$ \\
\hline \multirow{2}{*}{ Negativamente } & $\mathrm{n}$ & 5 & 11 & 8 & 24 & 1 & 2 & 0 & 3 \\
\hline & $\%$ & $13,9 \%$ & $17,7 \%$ & $21,1 \%$ & $17,6 \%$ & $2,9 \%$ & $3,6 \%$ & $0,0 \%$ & $2,4 \%$ \\
\hline \multirow{2}{*}{ Total } & $\mathrm{n}$ & 36 & 62 & 38 & 136 & 35 & 55 & 33 & 123 \\
\hline & $\%$ & $100 \%$ & $100 \%$ & $100 \%$ & $100 \%$ & $100 \%$ & $100 \%$ & $100 \%$ & $100 \%$ \\
\hline
\end{tabular}

Por otro lado, los padres consideran que las tecnologías digitales no tienen tanta influencia en el crecimiento personal o las relaciones sociales de los niños y adolescentes, presentándose ligeras diferencias entre los más jóvenes de la muestra y los más viejos.

Tabla 11. Influencia del uso de tecnología en crecimiento personal y relaciones sociales de su hijo según edad de los padres

\begin{tabular}{|c|c|c|c|c|c|c|c|c|c|}
\hline & & \multicolumn{4}{|c|}{ Crecimiento personal de su hijo } & \multicolumn{4}{|c|}{ Relaciones sociales de su hijo } \\
\hline \multirow{2}{*}{\multicolumn{2}{|c|}{ Tipo de influencia }} & \multicolumn{3}{|c|}{ Edad de los padres } & \multirow[b]{2}{*}{ Total } & \multicolumn{3}{|c|}{ Edad de los padres } & \multirow[b]{2}{*}{ Total } \\
\hline & & 23 a 32 & 33 a 42 & 43 años 0 & & 23 a 32 & 33 a 42 & 43 años 0 & \\
\hline \multirow{2}{*}{ Positivamente } & $\mathrm{n}$ & 10 & 24 & 12 & 46 & 9 & 19 & 12 & 40 \\
\hline & $\%$ & $30,3 \%$ & $44,4 \%$ & $32,4 \%$ & $37,1 \%$ & $25,7 \%$ & $35,2 \%$ & $34,3 \%$ & $32,3 \%$ \\
\hline \multirow{2}{*}{ No influye } & $\mathrm{n}$ & 17 & 24 & 17 & 58 & 16 & 24 & 14 & 54 \\
\hline & $\%$ & $51,5 \%$ & $44,4 \%$ & $45,9 \%$ & $46,8 \%$ & $45,7 \%$ & $44,4 \%$ & $40,0 \%$ & $43,5 \%$ \\
\hline \multirow{2}{*}{ Negativamente } & $\mathrm{n}$ & 6 & 6 & 8 & 20 & 10 & 11 & 9 & 30 \\
\hline & $\%$ & $18,2 \%$ & $11,1 \%$ & $21,6 \%$ & $16,1 \%$ & $28,6 \%$ & $20,4 \%$ & $25,7 \%$ & $24,2 \%$ \\
\hline \multirow{2}{*}{ Total } & $\mathrm{n}$ & 33 & 54 & 37 & 124 & 35 & 54 & 35 & 124 \\
\hline & $\%$ & $100 \%$ & $100 \%$ & $100 \%$ & $100 \%$ & $100 \%$ & $100 \%$ & $100 \%$ & $100 \%$ \\
\hline
\end{tabular}

Los padres perciben que la influencia de la tecnología contribuye al ejercicio de los derechos de sus hijos en relación con informarse, expresar su opinión, educarse y aprender por igual o sobre el 85\% (tabla 12). También consideran que contribuye positivamente al derecho de expresar su opinión, asociarse y manifestar su identidad personal sobre el $60 \%$ (tabla 13). No se presentan mayores diferencias por edades en el derecho a informarse y expresar su opinión. Sin embargo, la proporción de la percepción de influencia positiva es menor para el derecho a educarse y aprender, de asociarse y manifestar su identidad personal para las personas de mayor edad en la muestra.

Tabla 12. Influencia de uso de tecnología en derecho a informarse y a educarse y aprender según edad de los padres

\begin{tabular}{|c|c|c|c|c|c|c|c|c|c|}
\hline & & \multicolumn{4}{|c|}{ Derecho a informarse } & \multicolumn{4}{|c|}{ Derecho a educarse y aprender } \\
\hline \multirow{2}{*}{\multicolumn{2}{|c|}{ Tipo de contribución }} & \multicolumn{3}{|c|}{ Edad de los padres } & \multirow[b]{2}{*}{ Total } & \multicolumn{3}{|c|}{ Edad de los padres } & \multirow[b]{2}{*}{ Total } \\
\hline & & $\begin{array}{c}23 \text { a } 32 \\
\text { años }\end{array}$ & $\begin{array}{c}33 \text { a } 42 \\
\text { años }\end{array}$ & $\begin{array}{l}43 \text { años o } \\
\text { más }\end{array}$ & & $\begin{array}{c}23 \text { a } 32 \\
\text { años }\end{array}$ & $\begin{array}{c}33 \text { a } 42 \\
\text { años }\end{array}$ & $\begin{array}{c}43 \text { años o } \\
\text { más }\end{array}$ & \\
\hline \multirow{2}{*}{ Contribuye } & $\mathrm{n}$ & 33 & 54 & 32 & 119 & 35 & 50 & 27 & 112 \\
\hline & $\%$ & $91,7 \%$ & $91,5 \%$ & $91,4 \%$ & $91,5 \%$ & $92,1 \%$ & $84,7 \%$ & $79,4 \%$ & $85,5 \%$ \\
\hline \multirow{2}{*}{ No influye } & $n$ & 1 & 4 & 3 & 8 & 2 & 8 & 6 & 16 \\
\hline & $\%$ & $2,8 \%$ & $6,8 \%$ & $8,6 \%$ & $6,2 \%$ & $5,3 \%$ & $13,6 \%$ & $17,6 \%$ & $12,2 \%$ \\
\hline \multirow{2}{*}{ Obstaculiza } & $\mathrm{n}$ & 2 & 1 & 0 & 3 & 1 & 1 & 1 & 3 \\
\hline & $\%$ & $5,6 \%$ & $1,7 \%$ & $0,0 \%$ & $2,3 \%$ & $2,6 \%$ & $1,7 \%$ & $2,9 \%$ & $2,3 \%$ \\
\hline \multirow{2}{*}{ Total } & $\mathrm{n}$ & 36 & 59 & 35 & 130 & 38 & 59 & 34 & 131 \\
\hline & $\%$ & $100 \%$ & $100 \%$ & $100 \%$ & $100 \%$ & $100 \%$ & $100 \%$ & $100 \%$ & $100 \%$ \\
\hline
\end{tabular}


Tabla 13. Influencia de uso de tecnología en derecho a expresar su opinión, asociarse y manifestar identidad personal según edad de los padres

\begin{tabular}{|c|c|c|c|c|c|c|c|c|c|c|c|c|c|}
\hline & & \multicolumn{4}{|c|}{ Expresar su opinión } & \multicolumn{4}{|c|}{ Derecho a asociarse } & \multicolumn{4}{|c|}{ Manifestar la identidad personal } \\
\hline \multirow{2}{*}{\multicolumn{2}{|c|}{$\begin{array}{l}\text { Tipo de contribu- } \\
\text { ción }\end{array}$}} & \multicolumn{3}{|c|}{ Edad de los padres } & \multirow{3}{*}{$\begin{array}{c}\text { Total } \\
87 \\
\end{array}$} & \multicolumn{3}{|c|}{ Edad de los padres } & \multirow{3}{*}{$\begin{array}{c}\text { Total } \\
76\end{array}$} & \multicolumn{3}{|c|}{ Edad de los padres } & \multirow{3}{*}{\begin{tabular}{|c|} 
Total \\
73 \\
\end{tabular}} \\
\hline & & $\begin{array}{c}23 \text { a } 32 \\
\text { años }\end{array}$ & $\begin{array}{c}33 \text { a } 42 \\
\text { años }\end{array}$ & $\begin{array}{c}43 \\
\text { años o } \\
\text { más }\end{array}$ & & $\begin{array}{c}23 \text { a } 32 \\
\text { años }\end{array}$ & $\begin{array}{c}33 \text { a } 42 \\
\text { años }\end{array}$ & $\begin{array}{c}43 \\
\text { años o } \\
\text { más }\end{array}$ & & $\begin{array}{c}23 \text { a } 32 \\
\text { años }\end{array}$ & $\begin{array}{c}33 \text { a } 42 \\
\text { años }\end{array}$ & $\begin{array}{c}43 \\
\text { años o } \\
\text { más }\end{array}$ & \\
\hline \multirow{2}{*}{ Contribuye } & $\mathrm{n}$ & 26 & 38 & 23 & & 26 & 33 & 17 & & 23 & 31 & 19 & \\
\hline & $\%$ & $72,2 \%$ & $70,4 \%$ & $69,7 \%$ & $70,7 \%$ & $72,2 \%$ & $62,3 \%$ & $54,8 \%$ & $63,3 \%$ & $65,7 \%$ & $58,5 \%$ & $57,6 \%$ & $60,3 \%$ \\
\hline \multirow{2}{*}{ No influye } & $\mathrm{n}$ & 10 & 14 & 7 & 31 & 8 & 17 & 8 & 33 & 10 & 18 & 10 & 38 \\
\hline & $\%$ & $27,8 \%$ & $25,9 \%$ & $21,2 \%$ & $25,2 \%$ & $22,2 \%$ & $32,1 \%$ & $25,8 \%$ & $27,5 \%$ & $28,6 \%$ & $34,0 \%$ & $30,3 \%$ & $31,4 \%$ \\
\hline \multirow{2}{*}{ Obstaculiza } & $\mathrm{n}$ & 0 & 2 & 3 & 5 & 2 & 3 & 6 & 11 & 2 & 4 & 4 & 10 \\
\hline & $\%$ & $0,0 \%$ & $3,7 \%$ & $9,1 \%$ & $4,1 \%$ & $5,6 \%$ & $5,7 \%$ & $19,4 \%$ & $9,2 \%$ & $5,7 \%$ & $7,5 \%$ & $12,1 \%$ & $8,3 \%$ \\
\hline \multirow{2}{*}{ Total } & $\mathrm{n}$ & 36 & 54 & 33 & 123 & 36 & 53 & 31 & 120 & 35 & 53 & 33 & 121 \\
\hline & $\%$ & $100 \%$ & $100 \%$ & $100 \%$ & $100 \%$ & $100 \%$ & $100 \%$ & $100 \%$ & $100 \%$ & $100 \%$ & $100 \%$ & $100 \%$ & $100 \%$ \\
\hline
\end{tabular}

Los encuestados perciben que el tipo de influencia de la tecnología en la comunicación dependerá de con quién se comunique su hijo. Se observa una percepción de influencia negativa de la tecnología en la comunicación entre los niños y sus padres y en relación con otra persona mayor (tabla 14) existiendo diferencias según la edad de los padres. Por un lado, los más jóvenes consideran en mayor proporción que la tecnología influye negativamente en la comunicación de los hijos con sus padres. Mientras es mayor la proporción del grupo de más edad de la muestra que percibe la influencia negativa de la tecnología en la relación con personas mayores que sus hijos.

Tabla 14. Influencia de uso de tecnología en comunicación de los hijos con sus padres y relación con personas mayores que sus hijos según la edad de los padres

\begin{tabular}{|c|c|c|c|c|c|c|c|c|c|}
\hline & & \multicolumn{4}{|c|}{ Comunicación con sus padres } & \multicolumn{4}{|c|}{ Relación con personas mayores que sus hijos } \\
\hline \multirow{2}{*}{\multicolumn{2}{|c|}{ Tipo de influencia }} & \multicolumn{3}{|c|}{ Edad de los padres } & \multirow[b]{2}{*}{ Total } & \multicolumn{3}{|c|}{ Edad de los padres } & \multirow[b]{2}{*}{ Total } \\
\hline & & $\begin{array}{c}23 \text { a } 32 \\
\text { años }\end{array}$ & $\begin{array}{c}33 \text { a } 42 \\
\text { años }\end{array}$ & $\begin{array}{l}43 \text { años o } \\
\text { más }\end{array}$ & & $\begin{array}{c}23 \text { a } 32 \\
\text { años }\end{array}$ & $\begin{array}{c}33 \text { a } 42 \\
\text { años }\end{array}$ & $\begin{array}{l}43 \text { años o } \\
\text { más }\end{array}$ & \\
\hline \multirow{2}{*}{ Positivamente } & $\mathrm{n}$ & 9 & 15 & 12 & 36 & 1 & 6 & 5 & 12 \\
\hline & $\%$ & $23,7 \%$ & $25,9 \%$ & $34,3 \%$ & $27,5 \%$ & $2,8 \%$ & $11,5 \%$ & $15,6 \%$ & $10,0 \%$ \\
\hline \multirow{2}{*}{ No Influye } & $\mathrm{n}$ & 10 & 22 & 10 & 42 & 12 & 17 & 6 & 35 \\
\hline & $\%$ & $26,3 \%$ & $37,9 \%$ & $28,6 \%$ & $32,1 \%$ & $33,3 \%$ & $32,7 \%$ & $18,8 \%$ & $29,2 \%$ \\
\hline \multirow{2}{*}{ Negativamente } & $\mathrm{n}$ & 19 & 21 & 13 & 53 & 23 & 29 & 21 & 73 \\
\hline & $\%$ & $50,0 \%$ & $36,2 \%$ & $37,1 \%$ & $40,5 \%$ & $63,9 \%$ & $55,8 \%$ & $65,6 \%$ & $60,8 \%$ \\
\hline \multirow{2}{*}{ Total } & $\mathrm{n}$ & 38 & 58 & 35 & 131 & 36 & 52 & 32 & 120 \\
\hline & $\%$ & $100 \%$ & $100 \%$ & $100 \%$ & $100 \%$ & $100 \%$ & $100 \%$ & $100 \%$ & $100 \%$ \\
\hline
\end{tabular}

Por el contrario, se observa que los padres perciben que la tecnología no tiene influencia en la relación con niños menores que sus hijos, no identificándose diferencias entre los grupos de edad de la muestra (tabla 15). En cuanto a la relación que pueden tener sus hijos con otros niños de su edad, los padres perciben que no existen mayores diferencias entre los tipos de influencia. Mientras los más jóvenes consideran en mayor proporción que la tecnología no tiene influencia, los mayores consideran que tienen una influencia positiva.

Tabla 15. Influencia de uso de tecnología en relación con personas de su edad y en relación con personas menores que ellos según edad de los padres

\begin{tabular}{|c|c|c|c|c|c|c|c|c|c|}
\hline & & \multicolumn{4}{|c|}{ Relación con personas de su edad } & \multicolumn{4}{|c|}{ Relación con personas menores que ellos } \\
\hline \multirow{2}{*}{\multicolumn{2}{|c|}{ Tipo de influencia }} & \multicolumn{3}{|c|}{ Edad de los padres } & \multirow{2}{*}{ Total } & \multicolumn{3}{|c|}{ Edad de los padres } & \multirow[b]{2}{*}{ Total } \\
\hline & & 23 a 32 & 33 a 42 & 43 años 0 & & 23 a 32 & 33 a 42 & 43 años o & \\
\hline \multirow{2}{*}{ Positivamente } & $\mathrm{n}$ & 10 & 16 & 13 & 39 & 4 & 3 & 5 & 12 \\
\hline & $\%$ & $27,8 \%$ & $30,2 \%$ & $41,9 \%$ & $32,5 \%$ & $11,1 \%$ & $6,0 \%$ & $15,6 \%$ & $10,2 \%$ \\
\hline \multirow{2}{*}{ No influye } & $\mathrm{n}$ & 15 & 20 & 8 & 43 & 23 & 33 & 19 & 75 \\
\hline & $\%$ & $41,7 \%$ & $37,7 \%$ & $25,8 \%$ & $35,8 \%$ & $63,9 \%$ & $66,0 \%$ & $59,4 \%$ & $63,6 \%$ \\
\hline \multirow{2}{*}{ Negativamente } & $\mathrm{n}$ & 11 & 17 & 10 & 38 & 9 & 14 & 8 & 31 \\
\hline & $\%$ & $30,6 \%$ & $32,1 \%$ & $32,3 \%$ & $31,7 \%$ & $25,0 \%$ & $28,0 \%$ & $25,0 \%$ & $26,3 \%$ \\
\hline \multirow{2}{*}{ Total } & $\mathrm{n}$ & 36 & 53 & 31 & 120 & 36 & 50 & 32 & 118 \\
\hline & $\%$ & $100 \%$ & $100 \%$ & $100 \%$ & $100 \%$ & $100 \%$ & $100 \%$ & $100 \%$ & $100 \%$ \\
\hline
\end{tabular}


El análisis permitió revelar aspectos a profundizar que se están trabajando actualmente en una segunda fase cualitativa de entrevistas en profundidad y grupos focales con padres, madres y profesores.

\section{Discusión. Padres ante un nuevo desafío: la alfabetización transmedia}

De acuerdo con la tasa de respuesta femenina en las encuestas $(81,7 \%)$, se tiene una mayor representación de las madres que de los padres. En general en Chile las madres están más presentes en el cuidado e involucramiento en la vida escolar de sus hijos. En este sentido, las prácticas de mediación parental son determinadas mayormente por mujeres y sustentadas por las percepciones que estas tienen de las TIC. Por esa razón, es importante que las políticas públicas de alfabetización transmedia para

En general en Chile las madres están más presentes en el cuidado e involucramiento en la vida escolar de sus hijos adultos consideren especialmente a las mujeres, quie-

nes además tienen que superar brechas, pues han sido vistas de forma prejuiciosa en Chile en cuanto a su vínculo con las nuevas tecnologías (Phillippi; Peña, 2015). Asimismo resulta clave diseñar estrategias para que los padres asuman y desempeñen el nuevo reto de guiar a sus hijos en un mundo transmedia. La cuestión de la variedad de los perfiles tecnológicos y cómo estos podrían asociarse con las percepciones sobre las TIC y las prácticas de mediación son elementos importantes para ser incluidos en planes de formación de apoderados.

Los resultados de esta primera parte de la investigación demuestran la vigencia y actualidad de la sociedad red (Castells, 2006). Los encuestados informan que realizan varias actividades en internet, independientemente de la edad que tengan. Aunque sí existe una diferencia en cuanto a la cantidad de horas que se encuentran conectados, las personas mayores pasan menos tiempo en internet, mientras que los jóvenes están conectados varias horas más.

En cuanto a las diferentes competencias que incluye la alfabetización transmedia ya reseñadas, los encuestados coinciden en que el uso de las nuevas tecnologías influye positivamente en la educación de sus hijos en un entorno digital. De la misma forma señalan, independiente de su edad, que su uso influye positivamente en el ejercicio de los Derechos de los Niños que reconoce la Convención de Unicef.

Cuando analizamos cuál es la percepción de los padres sobre el uso de la tecnología en varios aspectos de la vida de sus hijos surge una situación interesante de profundizar en la fase cualitativa posterior:

- desde una posición más normativa, afirmaron que el uso de las TIC influía positivamente en el desempeño escolar de sus hijos, en su futuro universitario y laboral;

- al mismo tiempo, creen que no influyen en el crecimiento personal de los pequeños y en las relaciones sociales que establecen con otros, lo que se contradice con el alto porcentaje que reconoce la importancia del uso de internet para el ejercicio de los derechos de los niños.

Siguiendo la misma línea, encontramos una paradoja. Para los padres el uso de las tecnologías digitales influye negativamente en que los niños se relacionen con personas mayores que ellos, pero no influye en que lo hagan con personas menores que ellos mismos. También llama la atención el hecho de que señalaron que el uso de las tecnologías influye negativamente en la comunicación que establecen con sus hijos. Vale la pena subrayar que los encuestados reconocieron emplear las pantallas como recurso de regulación de las relaciones de autoridad con sus hijos (recompensa o desincentivo; castigo- para premiar o anular conductas que ellos consideren inadecuadas), aspecto en el que también habría que profundizar, pues además se contradice con la formación de ciudadanía desde los derechos a la comunicación. Más allá de una necesidad de alfabetización transmedia, esta situación nos interpela desde una perspectiva cultural, en cuanto a cuán legítimo se sigue considerando el castigo en el contexto de la Convención internacional de los derechos de los niños de Naciones Unidas.

Los padres más jóvenes son los que comprueban con mayor frecuencia el uso que hacen sus hijos de las TIC, quizás porque son ellos quienes más tiempo pasan conectados. También son los que menos piden a sus hijos que los ayuden y enseñen a usar las TIC.

Conocer las prácticas de mediación parental y las percepciones que las sustentan ofrece luces para delinear el camino de la alfabetización transmedia dirigida a este grupo poblacional. En ese sentido, concluimos que es necesario diseñar estrategias que involucren a madres, padres y otros familiares adultos que puedan servir de guía a los pequeños en el complejo mundo transmedia que hoy vivimos.

También es necesario educar a los adultos en el uso de las TIC para lograr una comunicación asertiva con sus hijos, utilizando todas las herramientas que les ofrece el ámbito digital. En este caso resulta relevante además demostrar a los padres las potencialidades de las TIC más allá del escenario escolar y el futuro laboral de los pequeños, con el objetivo de que las reconozcan como un nuevo espacio de socialización y entretenimiento al alcance de sus hijos. 
Por último, señalamos la necesidad de educar a los padres en el uso de controles parentales para evitar que sus hijos estén expuestos a situaciones de riesgo en la Red. Asimismo es importante desarrollar en ellos habilidades relacionadas con la privacidad en el entorno digital, pues de esta forma estarían más tranquilos con respecto al acoso de personas mayores y podrían guiar de mejor manera a sus hijos para que actúen de forma adecuada en estos casos.

Por el momento, sólo nos queda entonces ampliar y profundizar en las distintas necesidades de alfabetismo transmedia que aquí emergieron. La fase cualitativa que se desarrollará próximamente como parte de este estudio también contribuirá a que los padres puedan acompañar responsablemente el crecimiento de sus hijos junto a las TIC, cumpliendo así con el desafío y su nuevo rol en el contexto de la sociedad digital.

\section{Referencias}

Aguaded, Ignacio (2011). "Niños y adolescentes. Nuevas generaciones interactivas". Comunicar, v. 18, n. 36, pp. 7-8. https://doi.org/10.3916/C36-2011-01-01

Aguaded, Ignacio (2012). “Apuesta de la ONU por una educación y alfabetización mediáticas”. Comunicar, v. 19, n. 38, pp. 7-8.

https://doi.org/10.3916/C38-2012-01-01

Aierbe, Ana A.; Medrano, Concepción; Orejudo, Santos (2008). "Hábitos televisivos, valores y mediación parental en adolescentes". Revista mexicana de psicología, v. 25, n. 2, pp. 259-270.

http://www.redalyc.org/articulo.oa? $\mathrm{id}=243016308006$

Aparici, Roberto; García-Marín, David (2017). Comunicar y educar en el mundo que viene. Barcelona: Gedisa. ISBN: 978 8416919642

Area-Moreira, Manuel (2010). "Tecnologías digitales, multialfabetización y bibliotecas de la escuela del siglo XXI". Boletín de la Asociación Andaluza de Bibliotecarios, v. 98/99, pp. 39-52.

https://dialnet.unirioja.es/servlet/articulo?codigo $=3616424$

Aufderheide, Patricia (1993). Media literacy. A report of the National Leadership Conference on Media Literacy. Maryland, Estados Unidos: Aspen Institute. ISBN: 0898431379

https://files.eric.ed.gov/fulltext/ED365294.pdf

Bartau-Rojas, Isabel; Aierbe-Barandiaran, Ana; Oregui-González, Eider (2018). “Mediación parental del uso de Internet en el alumnado de primaria: creencias, estrategias y dificultades”. Comunicar, v. 24, n. 54, pp. 71-79.

https://doi.org/10.3916/C54-2018-07

Buckingham, David (2005). The media literacy of children and young people. A review of the research literature on behalf of Ofcom. London: Centre for the Study of Children Youth and Media Institute of Education, University of London. https://pdfs.semanticscholar.org/26b8/00cf297423784519edddacfde6a516d5872f.pdf

Buckingham, David (2009). "The future of media literacy in the digital age: some challenges for policy and practice". Medien impulse, v. 47, n. 2.

http://medienimpulse.erz.univie.ac.at/articles/view/143

Bringué-Sala, Xavier; Sádaba-Chalezquer, Charo (coord.) (2008). La generación interactiva en Iberoamérica. Niños y adolescentes frente a las pantallas. Madrid: Ariel; Fundación Telefónica. ISBN: 9788408084549

http://www.frra.utn.edu.ar/fae/docs/Generaciones/nteractivas.pdf

Bringué-Sala, Xavier; Sádaba-Chalezquer, Charo; Tolsà-Caballero, Jorge (2010). La generación interactiva en Iberoamérica 2010. Niños y adolescentes ante las pantallas. Madrid: Fundación Telefónica. ISBN: 97880812141

Bruns, Axel (2008). "The future is user-led: the path towards widespread produsage". In: PerthDAC 2007 Conference (Perth, 16 October, 2017).

http://produsage.org/node/15

Castells, Manuel (2006). La sociedad red: una visión global. Madrid: Alianza Editorial. ISBN: 9788420647845

Castells, Manuel (2007). “Estudiar, ¿ para qué?”. La vanguardia, 24 noviembre.

Chiavassa, Antonio (2015). "Carlos Scolari: la hora del prosumidor". El cactus, v. 4, n. 4, pp. 24-26.

https://revistas.unc.edu.ar/index.php/Cactus/article/viewFile/13103/13298

Ciastellardi, Matteo; Di-Rosario, Giovanna (2015). "Transmedia literacy. A premise”. International journal of transmedia literacy, v. 1, n. 1, pp. 7-16. 
https://doi.org/10.7358/ijtl-2015-001-cias

Comisión Europea (2007). A European approach to media literacy in the digital environment. Commission of the European Communities.

http://eurlex.europa.eu/LexUriServ/LexUriServ.do?uri=COM\%3A2007\%3A0833\%3AFIN\%3AEN\%3APDF

Condeza, Rayén; De-Fontcuberta, Mar (2015). "Redes sociales: Luces y sombras en el ámbito de la comunicación y educación". En: Tomé, Vitor; Bévort, Evelyne; Reia-Baptista, Vitor (eds.). Research on social media: a glocal view/ Investigação em media sociais: uma visão glocal. Algarve: RVJ. Science and Technology Portuguese Foundation-Ciac, Algarve University, pp. 51-70. ISBN: 9789898289452

Costa, Conceição (2011). "Why does society need media literacy?”. In: Livingstone, Sonia (ed.). Media literacy: Ambitions, policies and measures. Reino Unido: Cost, LSE, pp. 26-30. ISBN: 9782960115727

De-Fontcuberta, Mar; Guerrero, Claudio (2011). "Una nueva propuesta para la educación en medios". Cuadernos.info, v. 20, pp. 87-97.

https://doi.org/10.7764/cdi.20.115

Dudaceck, Oto (2015). "Transmedia storytelling in education". Social and behavioral sciences, v. 197, pp. $694-696$. https://doi.org/10.1016/j.sbspro.2015.07.062

Ferrés-Prats, Joan (2010). "Educomunicación y cultura participativa". En: Aparici, Roberto (coord.). Educomunicación: más allá del 2.0. Barcelona: Gedisa, pp. 251-266. ISBN: 9788497846059

Frau-Meigs, Divina (2012). "Transliteracy as the new research horizon for media and information literacy". Medijske studije, v. 3, n. 6, pp. 14-26.

https://hrcak.srce.hr/ojs/index.php/medijske-studije/article/view/6064

García-Ruiz, Rosa; Ramírez-García, Antonia; Rodríguez-Rossell, María-del-Mar (2014). “Educación en alfabetización mediática para una nueva ciudadanía prosumidora". Comunicar, v. 22, v. 43, pp. 15-23.

https://doi.org/10.3916/C43-2014-01

Jenkins, Henry; Ito, Mizuko; Boyd, Danah (2015). Participatory culture in a networked era. A conversation on youth, learning, commerce and politics. Cambridge: Polity Press. ISBN: 9780745660714

Koltay, Tibor (2011). "The media and the literacies: Media literacy, information literacy, digital literacy". Media culture and society, v. 33, n. 2, pp. 211-221.

https://doi.org/10.1177/0163443710393382

Livingstone, Sonia (2004). "Media literacy and the challenge of new information and communication technologies". Communication review, n. 7, v. 1, pp. 3-14.

https://doi.org/10.1080/10714420490280152

Livingstone, Sonia (ed.) (2011). Media literacy: Ambitions, policies and measures. Reino Unido: Cost, LSE. ISBN: 9782 960115727

Livingstone, Sonia (2016). "Reframing media effects in terms of children's rights in the digital age". Journal of children and media, v. 10, n. 1, pp. 4-12.

https://doi.org/10.1080/17482798.2015.1123164

Livingstone, Sonia; Haddon, Leslie (eds.) (2009). Kids online. Opportunities and risks for children. Bristol: The Policy Press. ISBN: 9781847424396

Livingstone, Sonia; Van-Couvering, Elizabeth; Thumim, Nancy (2005). Adult media literacy. A review of the research literature on behalf of Ofcom. Londres: Centre for the Study of Children Youth and Media Institute of Education, University of London.

https://dera.ioe.ac.uk/5283/1/aml.pdf

Marta-Lazo, Carmen; Gabelas-Barroso, José-Antonio (2008). "La familia en el escenario de las multipantallas". Doxa comunicación, v. 7, pp. 109-125.

https://repositorioinstitucional.ceu.es/bitstream/10637/5916/1/n\%c2\%baVII_pp109_125.pdf

Mihailidis, Paul; Viotty, Samantha (2017). "Spreadable spectacle in digital culture: civic expression, fake news and the role of media literacies in 'post-fact' societies". American behavioural scientist, v. 61, n. 4, pp. 441-454.

https://doi.org/10.1177/0002764217701217

Mineduc (2014). Estadísticas de la educación 2013. Ministerio de Educación de Chile. https://centroestudios.mineduc.cl/wp-content/uploads/sites/100/2017/07/Anuario_2013.pdf

Morduchowicz, Roxana (coord.) (2008). Los jóvenes y las pantallas. Nuevas formas de sociabilidad. Barcelona: Gedisa. ISBN: 9788497842693 
Nathanson, Amy I. (1999). “Identifying and explaining the relationship between parental mediation and children's agression". Communication Research, v. 26, n. 2, v. 124-143

https://doi.org/10.1177/009365099026002002

Orozco, Guillermo; Franco, Darwin (2014). Al filo de las pantallas. Buenos Aires: La Crujía. ISBN: 9789876012324

Padilla-Walker, Laura M.; Coyne, Sarah M. (2011). "'Turn that thing off!' parent and adolescent predictors of proactive media monitoring". Journal of adolescence, v. 34, pp. 705-715.

https://doi.org/10.1016/j.adolescence.2010.09.002

Pereira, Sara; Pinto, Manuel; Pereira, Luis (2012). “Recursos para la alfabetización mediática: investigación y propuestas para niños". Comunicar, v. 20, n. 39, pp. 91-99.

https://doi.org/10.3916/C39-2012-02-09

Phillippi, Alejandra; Peña, Patricia (2015). "The impact of public access to telecenters: Social appropriation of ICT by Chilean women". In: Proenza, Francisco J. (ed). Public access ICT across cultures. Diversifying participation in the network society. Ottawa: The MIT Press \& International Development Research Center (IDRC), Canada, pp. 315-340.

https://idl-bnc-idrc.dspacedirect.org/bitstream/handle/10625/54174/IDL-54174.pdf

Potter, James W. (2010). "The state of media literacy". Journal of broadcasting \& electronic media, v. 54, n. 4, pp. 675-696. https://doi.org/10.1080/08838151.2011.521462

Sádaba-Chalezquer, Charo; Bringué-Sala, Xavier (2010). “Niños y adolescentes españoles ante las pantallas: rasgos configuradores de una generación interactiva”. Participación educativa, n. 15, pp. 86-104.

https://dadun.unav.edu/bitstream/10171/18443/1/n15-sadaba-chalezquer.pdf

Santiago-Campión, Raúl; Navaridas-Nalda, Fermín; Andía-Celaya, Luis-Alberto (2016). “Las percepciones de los directivos escolares sobre el uso y el valor de las TIC para el cambio e innovación educativa". Estudios sobre educación, v. 30, pp. 145-174. https://doi.org/10.15581/004.30.145-174

Schofield-Clarck, Lynn (2011). "Parental mediation theory for the digital age". Communication theory, v. 21, n. 4, pp. 323-343. https://doi.org/doi:10.1111/j.1468-2885.2011.01391.x

Scolari, Carlos (2016). "Alfabetismo transmedia: estrategias de aprendizaje informal y competencias mediáticas en la nueva ecología de la comunicación. Telos, cuadernos de comunicación e innovación, v. 103, pp. 12-23.

https://repositori.upf.edu/handle/10230/27788?locale-attribute=es

Torreccillas-Lacave, Teresa; Vásquez-Barrio, Tamara; Monteagudo-Barandalla, Laura (2017). "Percepción de los padres sobre el empoderamiento digital de las familias en hogares hiperconectados". El profesional de la información, v. 26, n. 1, pp. 97-104.

https://doi.org/10.3145/epi.2017.ene.10

Unesco (2018). Media information literacy.

http://www.unesco.org/new/en/communication-and-information/media-development/media-literacy/mil-as-composite-concept

Winocur, Rosalía (2002). Ciudadanos mediáticos: la construcción de lo público en la radio. Barcelona: Gedisa. ISBN: 978 8474329308

Winocur, Rosalía (2007). “Nuevas tecnologías y usuarios. La apropiación de las TIC en la vida cotidiana". Telos: Cuadernos de comunicación, tecnología y sociedad, v. 73, pp.109-117.

https://dialnet.unirioja.es/servlet/articulo?codigo $=2487968$

Winocur, Rosalía (2009). Robinson Crusoe ya tiene celular: la conexión como espacio de control de la incertidumbre. México: Siglo XXI. ISBN: 9786070301193

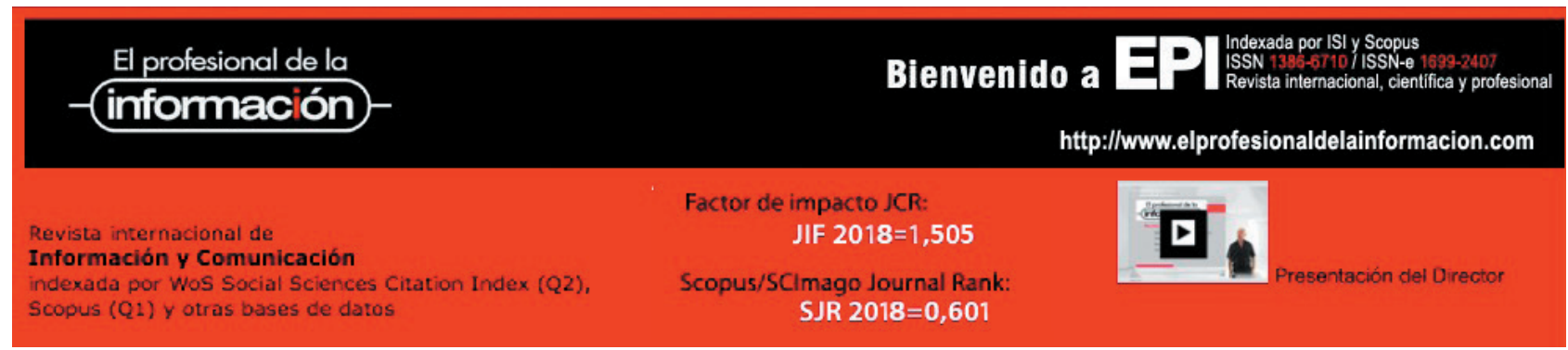

